\title{
USING A MICRO-UAV FOR ULTRA-HIGH RESOLUTION MULTI-SENSOR OBSERVATIONS OF ANTARCTIC MOSS BEDS
}

\author{
Arko Lucieer, Sharon Robinson, Darren Turner, Steve Harwin, and Josh Kelcey \\ School of Geography and Environmental Studies, Surveying and Spatial Sciences Group \\ University of Tasmania \\ Private Bag 76, Hobart, Tasmania 7001, Australia \\ Arko.Lucieer@utas.edu.au \\ http://www.terraluma.net
}

Commission ICWG I/V: Unmanned Vehicle Systems (UVS) for Mapping and Monitoring Applications

KEY WORDS: Unmanned Aerial Vehicle (UAV), Antarctica, moss beds, Structure from Motion (SfM) point cloud, terrain mapping, multispectral imagery

\begin{abstract}
:
This study is the first to use an Unmanned Aerial Vehicle (UAV) for mapping moss beds in Antarctica. Mosses can be used as indicators for the regional effects of climate change. Mapping and monitoring their extent and health is therefore important. UAV aerial photography provides ultra-high resolution spatial data for this purpose. We developed a technique to extract an extremely dense 3D point cloud from overlapping UAV aerial photography based on structure from motion (SfM) algorithms. The combination of SfM and patch-based multi-view stereo image vision algorithms resulted in a $2 \mathrm{~cm}$ resolution digital terrain model (DTM). This detailed topographic information combined with vegetation indices derived from a 6-band multispectral sensor enabled the assessment of moss bed health. This novel UAV system has allowed us to map different environmental characteristics of the moss beds at ultra-high resolution providing us with a better understanding of these fragile Antarctic ecosystems. The paper provides details on the different $\mathrm{UAV}$ instruments and the image processing framework resulting in DEMs, vegetation indices, and terrain derivatives.
\end{abstract}

\section{INTRODUCTION}

Polar regions are experiencing rapid and severe climatic shifts with major changes in temperature, wind speed and UV-B radiation already observed in Antarctica (Convey et al., 2009; Turner et al., 2005). Since vegetation is isolated to the coastal fringe and climatic records only extend back 50 years, with limited spatial resolution, we urgently need new proxies to determine if coastal climate has changed over the past century. In a manner similar to trees, old growth mosses also preserve a climate record along their shoots. Our ability to accurately date these mosses and map their extent in sufficient spatial detail means that, for the first time, mosses can be used as sentinels to provide crucial information on how the Antarctic coastal climate has changed over past centuries and how biota has responded to these changes (Lovelock and Robinson, 2002; Robinson et al., 2003; Wasley et al., 2006).

The spatial scale of the moss beds (tens of $\mathrm{m} 2$ ) makes satellite imagery (even recent very high resolution imagery of $0.5 \mathrm{~m}$ ) unsuitable for mapping their extent in sufficient detail. Due to logistical constraints aerial photography is impractical and also does not provide the required spatial resolution. Recent developments in the use of unmanned aerial vehicles (UAVs) for remote sensing applications provide exciting new opportunities for ultra-high resolution mapping and monitoring of the environment. A recent special issue on UAVs highlights that this field has an increasing potential for remote sensing applications (Zhou et al., 2009). Rango et al. (2006) and Hardin and Jackson (2005) developed and used a UAV based on a remote controlled helicopter and a plane capturing $; 1 \mathrm{~cm}$ resolution colour photography for rangeland mapping and monitoring. Several recent studies have highlighted the benefit of UAVs for crop mapping and monitoring (Berni et al., 2009; Hunt Jr et al., 2010; Lelong et al., 2008; ZarcoTejada, 2008). Laliberte and Rango (2009); Dunford et al. (2009) demonstrated how UAV imagery can be used for mapping natural vegetation using geographic object-based image analysis (GEOBIA) techniques. Finally, Nagai et al. (2009) showed how multiple sensors (visible, near-infrared, and LiDAR) can collect very high resolution data simultaneously from a large UAV. The key advantages of UAVs platform are their ability to fill a niche with respect to spatial and temporal resolution. The imagery acquired from a UAV is at sub-decimetre or even centimetre resolution (i.e. hyperspatial) and UAV imagery can be flown on-demand making it possible to capture imagery frequently allowing for efficient monitoring (i.e. hypertemporal).

In this study, we developed a small UAV based on a multi-rotor helicopter carrying three different cameras: RGB, 6-band multispectral, and thermal infrared for cost-effective, efficient, and ultra-high resolution mapping of moss beds within an Antarctic Special Protected Area (ASPA) near Casey, Windmill Islands, Antarctica. This paper focuses on a new technique, Structure from Motion (SfM), for deriving very dense 3D point clouds from overlapping UAV photography.

\section{STUDY AREA}

The Windmill Islands region near Casey has the most extensive and well-developed vegetation in Eastern Antarctica (Figure 1). The vegetation communities in Antarctica and the sub-Antarctic are undergoing rapid change. Climate change is now recognised as occurring in the high latitudes rendering Antarctica one of the most significant baseline environments for the study of global climate change. Temperature, UV-B and changes in water availability have been identified as the three key factors that will change in the Antarctic regions with climate change. Despite this, there have been few long-term studies of the response of Antarctic vegetation to climate (Convey et al., 2009; Robinson et al., 2003).

Most focus of change in the Antarctic region has been on the Antarctic Peninsula where dramatic shifts in temperature of up 


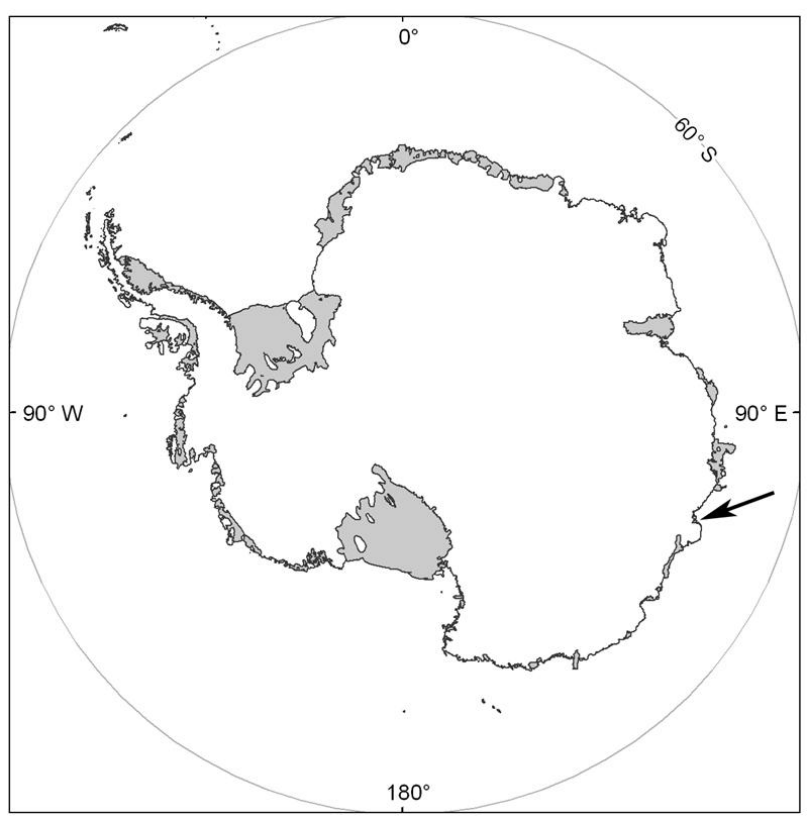

Figure 1: Study area: the continent of Antarctica with an arrow in Eastern Antarctica indicating the location of the Windmill Islands (source: Australian Antarctic Data Centre).

to 5 degrees Celsius have been recorded (Turner et al., 2005) with subsequent expansion of local plant communities. However, the first documented major change in a terrestrial community in Continental Antarctica has now been reported (King, 2009). Preliminary analysis of January 2008 data compared with 2003 data shows a decrease in live moss and a concomitant increase in moribund moss in Antarctic Specially Protected Area (ASPA) 135, Bailey Peninsula (King, 2009). Changes in colouration of moss turfs from green to red-brown have also been observed. This change in colouration is probably a stress response reflecting a transitional stage between live and moribund moss.

Our study site is the Robinson Ridge near Casey, one of Australia's Antarctic stations. The site is approximately $200 \mathrm{~m}$ by $100 \mathrm{~m}$ with several tens of $\mathrm{m}^{2}$ occupied by bryophytes (Figure 2 ). The location of bryophyte communities is largely driven by water availability and it is restricted to wet environments that receive water during the summer snow melt. Topographic factors, such as micro-topography (e.g. boulders and rocks), water drainage, upstream catchment, slope, and solar irradiance, play a key role in bryophyte occurrence and health.

\section{METHODS}

\subsection{UAV and sensors}

In this study, we used a multi-rotor helicopter, the OktoKopter developed by MikroKopter with a stabilised camera mount (Figure 3). The OktoKopter is capable of lifting $1.5 \mathrm{~kg}$ with a flight time between 6 and 10 minutes. The micro-UAV is generally guided by the on-board autopilot, however, due to the extreme magnetic compass declination and inclination we were unable to use the autopilot in Antarctica. We therefore flew the OktoKopter manually with a real-time downlink to the ground station to aid in the navigation. The flight path was recorded by a uBlox navigation-grade GPS receiver. The camera was stabilised by an active camera mount.



Figure 2: Moss bed area at the Robinson Ridge site with a variety of healthy moss (green), stressed moss (red/brown), and dead moss (black). Small orange discs (10 cm diameter) and trays ( 30 $\mathrm{cm}$ diameter) used as GCPs for geometric correction and validation are visible in the photograph.

The camera system consists of a Canon 550D DSLR, capturing 18 megapixel photographs every at approximately $1 \mathrm{~Hz}$. Photos were triggered by a custom-built cable connected to the OktoKopter's flight controller generate a $1 \mathrm{~Hz}$ pulse. We set the camera to aperture priority using the widest aperture and an ISO value between 200 and 800 to capture photographs at a shutter speed of $1 / 1600$ or faster in order to reduce motion blur. At a flying height of $50 \mathrm{~m}$ the $18 \mathrm{~mm}$ focal length lens captured aerial photographs with a spatial extent of $64 \mathrm{~m}$ by $33 \mathrm{~m}$ at $1 \mathrm{~cm}$ resolution. The time on the camera was synchronised with GPS time so that each photo could be geotagged afterwards. In addition, small orange markers were used as ground control point (GCPs) for accurate georegistration of the UAV photographs.

A second sensor was used to collect 6-band multispectral imagery. The Tetracam mini-MCA records six images of 1280x1024 pixels simultaneously. The band wavelengths were determined by $10 \mathrm{~nm}$ FWHM filters at the following wavelengths: 530, 550, $580,670,700$, and $800 \mathrm{~nm}$.

Two Leica GPS1200 receivers were used in dual frequency realtime kinematic mode, providing centimetre positional and height accuracies. Static GPS points were recorded for small aluminium markers that were installed during previous field seasons. These markers indicate the location of $20 \times 20 \mathrm{~cm}$ moss quadrats that have been photographed in the past and are used for photo monitoring and change detection. Accurately locating these moss quadrats will allow us to compare their locations to terrain layers and UAV photography in the future. In addition, 24 bright orange aluminium disks with a diameter of $10 \mathrm{~cm}$ were spread out over the study area. These disks act as ground control points (GCPs) with which the UAV aerial photographs were georeferenced. The influence of terrain (slope, solar radiation, hydrological conditions) on bryophyte health is very important. Differences in these terrain characteristics might explain the variability between the moss samples within a site and between sites. To determine these local environmental terrain characteristics a very high resolution digital elevation model (DEM) is required. The following section discusses how extremely dense point clouds $(1 \mathrm{~cm}$ point spacing) can be derived from overlapping UAV photography.

\subsection{Structure from Motion (SfM) point clouds}

Recently, a new computing vision technique, Structure from Motion (SfM), was introduced that allows the extraction of 3D structure of an object by analysing motion signals over time (Dellaert 


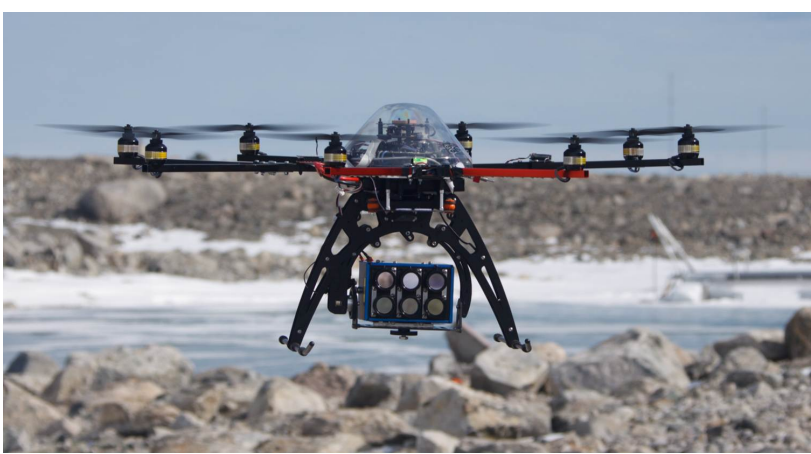

Figure 3: OktoKopter micro-UAV with 6-band multispectral sensor mounted on stabilised camera platform.

et al., 2000). The SfM technique can be applied to large collections of overlapping photographs to obtain sparse point clouds for a wide range of objects, such as buildings and sculptures (Snavely et al., 2006, 2007). The power of this technique was demonstrated by Snavely et al. (2007) who developed the Bundler software and used it to construct 3D models of well-known world sites, such as the Notre Dame, based on hundreds of overlapping photographs available from community websites. The technique is based on identifying matching features in images that are taken from different viewpoints. Image features are identified by the scale in variant feature transform (SIFT) algorithm (Lowe, 2004), which is robust in terms of its feature descriptors for image features at different viewing angles. The SfM algorithm also accommodates different cameras, lenses, focal points, and image resolutions. No prior knowledge is required on the position and orientation of the camera or lens distortion parameters. Once the SIFT features have been identified in each individual image, the features are matched against each other, using an approximate nearest neighbour (ANN) kd-tree approach, and poor matches are filtered out using RANSAC (Snavely et al., 2006, 2007). Based on these SIFT matches, the camera positions, orientations, radial lens distortion, and finally the 3D coordinates of each SIFT feature are calculated using a bundle block adjustment. The 3D positions of the SIFT features essentially form a 3D point cloud that captures the structure of an object.

The point cloud is known as a sparse point cloud that can be densified with a more recent technique called multi-view stereopsis (Furukawa and Ponce, 2009). The stereopsis algorithm takes the output from the Bundler algorithm, camera positions, orientations, and radial undistorted images, and applies a match, expand, and filter procedure. It starts with the sparse set of matched keypoints, and repeatedly expands these points to neighbouring pixel correspondences, and finally applies visibility constraints to filter out false matches (Furukawa and Ponce, 2009). The algorithm is implemented in the Patch View Multi-Stereo (PMVS2) software tool. SIFT, Bundler, and PMVS2 work in sequence to generate an extremely dense 3D point cloud just from overlapping photographs. The PMVS2 point cloud contains the following information for each point: XYZ coordinates, point normals (i.e. direction of the slope through the point), and point RGB colour values (i.e. derived from the photographs). Even though these computing vision algorithms were designed for building and object reconstruction, this study is the first to show that the algorithms work very well on UAV aerial photography. Movement of the UAV platform at low altitudes (e.g. $50100 \mathrm{~m}$ ) ensures that terrain features are captured from a wide range of angles. Our UAV camera setup allows frequent capture of aerial photographs, resulting in 100+ photographs for a typical $5 \mathrm{~min}$. flight covering $1 \mathrm{ha}$, which ensures that there is sufficient overlap for SfM

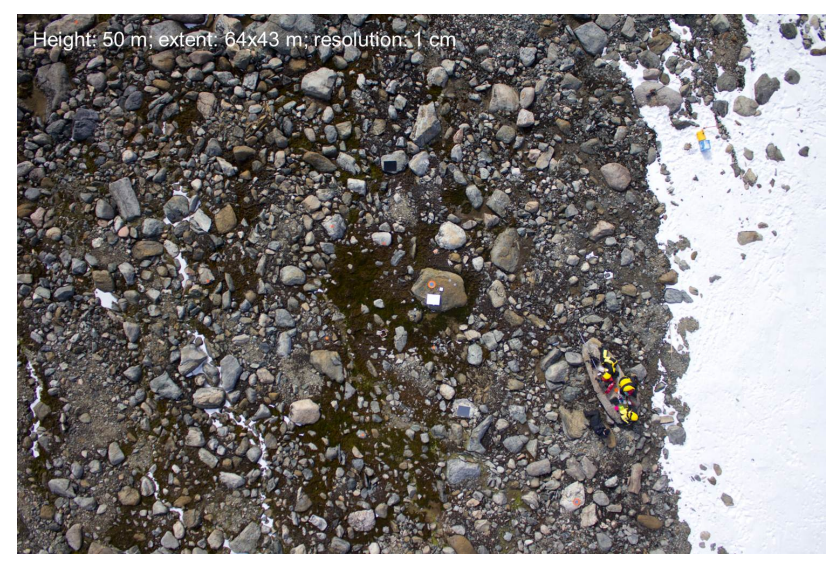

Figure 4: DSLR photograph for a section of moss bed.

algorithms to work efficiently and accurately.

One of the problems of using SfM point clouds in geographical applications is that the point clouds coordinates are in an arbitrary coordinate system. In order for the point cloud to be compatible with other spatial datasets it needs to be transformed to a real-world coordinate system. In this study, we used bright orange markers as ground control. The PMVS2 point cloud has an approximate point spacing of 1-2 $\mathrm{cm}$. This incredible point density allows each orange marker disc to be covered in multiple points. The marker points were filtered out of the point cloud based on their colour values and a user-defined RGB threshold. The marker points were then matched to the corresponding DGPS coordinates. A 7-parameter Helmert transformation was calculated based on the coordinate pairs (PMVS2 XYZ - GPS Easting, Northing, Height) and the transformation was applied to all points in the point cloud, resulting in a georeferenced point cloud. Finally, the point cloud coordinates with their RGB values were exported to the LAS 1.2 format, commonly used for LiDAR datasets. LiDAR visualisation and interpolation tools can then be used to explore and analyse the point cloud. A triangulation and rasterisation resulted in a $1 \mathrm{~cm}$ resolution DEM of the moss bed area. Several terrain derivatives, such as slope, topographic wetness index, and solar radiation were derived from the DEM. In future research, these terrain derivatives will be related to health and moisture information derived for each moss quadrat in order to explore the relationship between drying trends and environmental terrain parameters.

\section{RESULTS AND DISCUSSION}

Figure 4 shows a DSLR photograph acquired by the UAV flying at $50 \mathrm{~m}$ above ground level (AGL). The spatial resolution of the images is $1 \mathrm{~cm}$. An experimental plot can be seen just left of centre showing spectral calibration panels and orange disks for ground control (GCPs). The mosses show up as green and brown in amongst the rocks. The snow on the eastern side of the image feeds the moss beds through melt streams.

Figure 5 shows a point cloud of a 5 by $5 \mathrm{~m}$ area (e.g.the experimental plot in the centre of Figure 4) derived using Bundler and PMVS2. The point spacing is $1-2 \mathrm{~cm}$. The point cloud is converted to the LAS 1.2 format storing height, and RGB colour information for each point. The micro-topography is captured in great detail showing small rocks and relief in the mosses (caused by forst heave).

Figure 6 shows a triangulated surface derived from the PMVS2 point cloud with each triangle coloured according to the average 


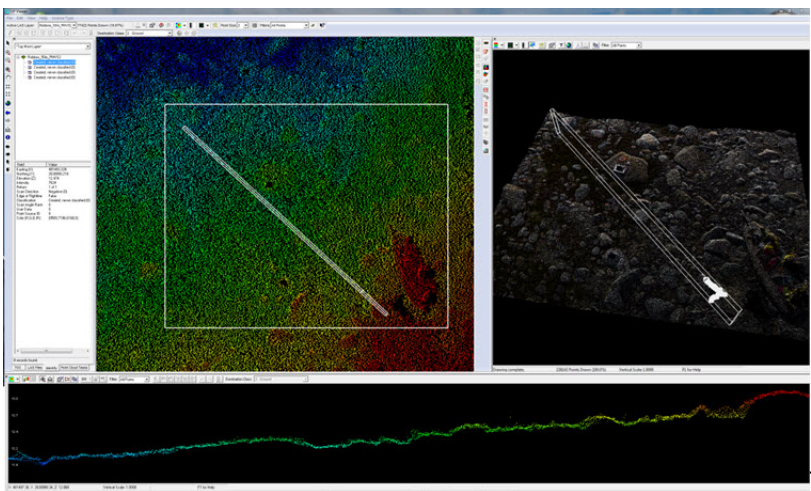

Figure 5: Point cloud showing the detailed micro-topography captured by the PMVS2 technique.

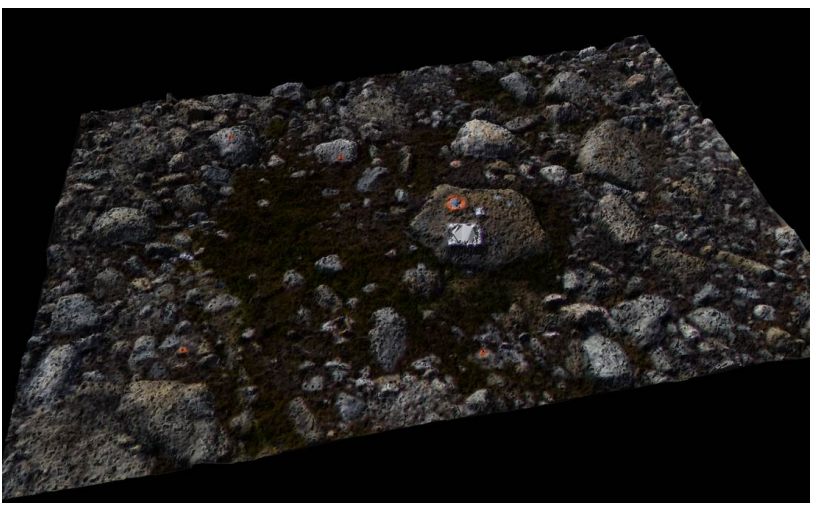

Figure 6: Terrain surface model derived from the point cloud.

colour in the overlapping photographs. This surface was converted to a digital elevation model at $2 \mathrm{~cm}$ grid cell resolution. From this DEM a topographic wetness index (TWI) was derived using a Monte Carlo simulation in SAGA GIS (Figure 7). The TWI highlights areas of potentially high water concentration as a function of upstream area and slope. It does not take into account soil characteristics or complex snow melt processes, however, the TWI could be a good proxy for identification of water availability.

Figure 8 shows a false colour composite of the experimental plot area. Healthy moss clearly show a high reflectance in near-infrared, highlighted by the red colour in this image. The normalised difference vegetation index (NDVI) derived from the NIR and red

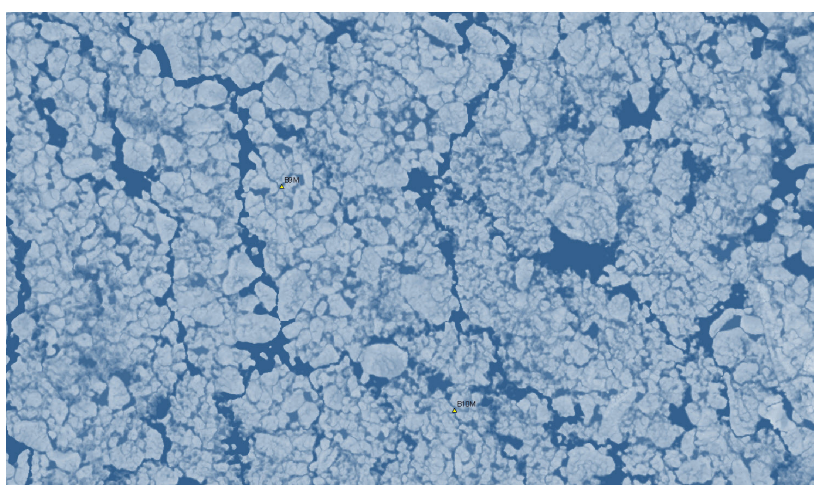

Figure 7: Topographic wetness index derived from the highresolution digital elevation model.

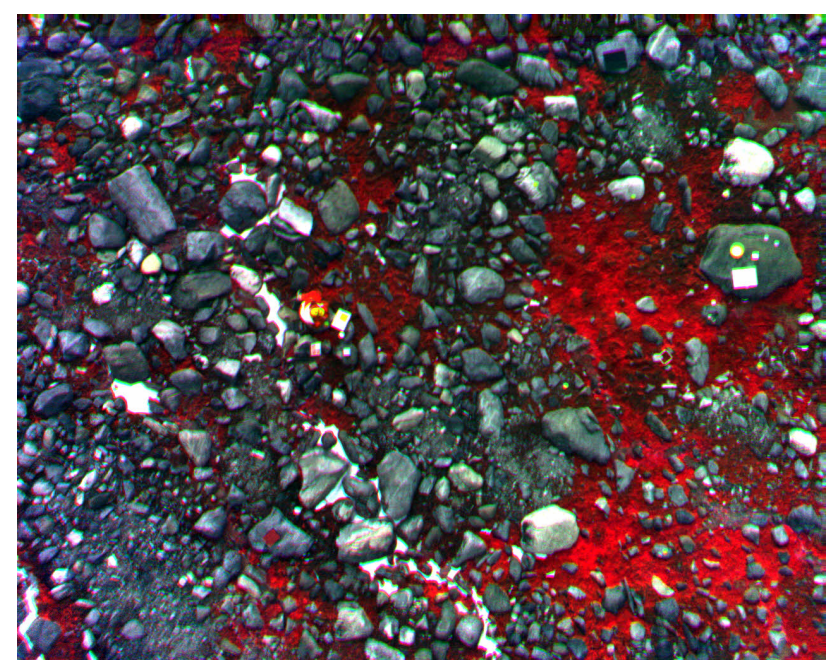

Figure 8: False colour composite $(\mathrm{RGB}=800,670,550 \mathrm{~nm})$ captured by the mini-MCA.

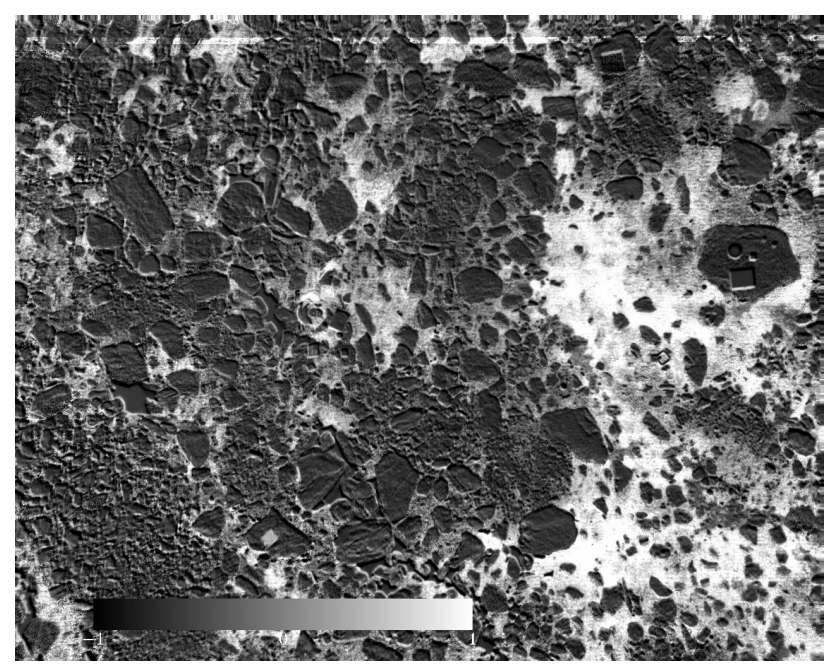

Figure 9: Normalised Difference Vegetation Index (NDVI) derived from the MCA imagery.

bands highlights the location and extent of healthy moss. In future research we aim to relate the spectral characteristics of moss to environmental indicators, such as the wetness index.

\section{CONCLUSIONS AND FUTURE WORK}

This study has demonstrated the application of an exciting new technique for 3D point cloud generation from overlapping UAV photography. Structure from Motion (SfM) algorithms designed for object reconstruction from overlapping photography were successfully applied to UAV photographs of an Antarctic moss bed, deriving extremely detailed 3D point clouds of the terrain. The point cloud was transformed to a real-world coordinate system based on ground control points with an accuracy of $3 \mathrm{~cm}$. The resulting point cloud had a point spacing of $1-2 \mathrm{~cm}$ covering the study area in six million points. Subsequent interpolation produced a $2 \mathrm{~cm}$ resolution DEM, capturing very fine details in the moss bed topography. These detailed terrain features will be used in future research to explore the relationship between moss dieback, caused by to water stress, and terrain characteristics.

Finally, we are currently developing a hyperspectral capable UAV. 
The integration of a mini-hyperspectral pushbroom scanner with an IMU/GPS sensor, data logger with accurate timestamping, and a UAV airframe will allow us to collect ultra-high resolution hyperspectral imagery. Preliminary field scans from the 2012 Summer season show promising results.

\section{ACKNOWLEDGEMENTS}

The authors would like to acknowledge the Australian Antarctic Division for financial and logistic support (project AAS313). We would also like to thank Dr Dana Bergstrom and Mrs Jessica Bramley-Alves for their support in the field.

\section{References}

Berni, J. A. J., Zarco-tejada, P. J., Suárez, L. and Fereres, E., 2009. Thermal and Narrowband Multispectral Remote Sensing for Vegetation Monitoring From an Unmanned Aerial Vehicle. IEEE Transactions on Geoscience and Remote Sensing 47(3), pp. 722-738.

Convey, P., Bindschadler, R., di Prisco, G., Fahrbach, E., Gutt, J., Hodgson, D., Mayewski, P., Summerhayes, C. and Turner, J., 2009. Antarctic climate change and the environment. Antarctic Science 21(06), pp. 541.

Dellaert, F., Seitz, S., Thorpe, C. and Thrun, S., 2000. Structure from motion without correspondence. In: Proceedings IEEE Conference on Computer Vision and Pattern Recognition. CVPR 2000 (Cat. No.PR00662), Vol. 26number 2, IEEE Comput. Soc, Hilton Head Island, SC , USA, pp. 557-564.

Dunford, R., Michel, K., Gagnage, M., Piegay, H. and Tremelo, M. L., 2009. Potential and constraints of Unmanned Aerial Vehicle technology for the characterization of Mediterranean riparian forest. International Journal of Remote Sensing 30(19), pp. 4915-4935.

Furukawa, Y. and Ponce, J., 2009. Accurate, Dense , and Robust Multi-View Stereopsis. In: IEEE Transactions on Pattern Analysis and Machine Intelligence, IEEE Computer Society Press.

Hardin, P. and Jackson, M., 2005. An unmanned aerial vehicle for rangeland photography. Rangeland Ecology \& Management 58(4), pp. 439-442.

Hunt Jr, E., Hively, W., Fujikawa, S., Linden, D., Daughtry, C. and McCarty, G., 2010. Acquisition of NIR-Green-Blue Digital Photographs from Unmanned Aircraft for Crop Monitoring. Remote Sensing 2(1), pp. 290-305.

King, D., 2009. Rapid change in continental Antarctic vegetation communities. Honours, University of Wollongong.

Laliberte, A. S. and Rango, A., 2009. Texture and Scale in Object-Based Analysis of Subdecimeter Resolution Unmanned Aerial Vehicle (UAV) Imagery. IEEE Transactions on Geoscience and Remote Sensing 47(3), pp. 761-770.

Lelong, C. C. D., Burger, P., Jubelin, G., Roux, B., Labbé, S. and Baret, F., 2008. Assessment of unmanned aerial vehicles imagery for quantitative monitoring of wheat crop in small plots. Sensors 8, pp. 3557-3585.

Lovelock, C. and Robinson, S., 2002. Surface reflectance properties of Antarctic moss and their relationship to plant species, pigment composition and photosynthetic function. Plant, Cell \& Environment 25(10), pp. 1239-1250.
Lowe, D. G., 2004. Distinctive Image Features from ScaleInvariant Keypoints. International Journal of Computer Vision 60(2), pp. 91-110.

Nagai, M., Chen, T., Shibasaki, R., Kumagai, H. and Ahmed, A., 2009. UAV-Borne 3-D Mapping System by Multisensor Integration. IEEE Transactions on Geoscience and Remote Sensing 47(3), pp. 701-708.

Rango, A., Laliberte, A., Steele, C., Herrick, J., Bestelmeyer, B., Schmugge, T., Roanhorse, A. and Jenkins, V., 2006. Using Unmanned Aerial Vehicles for Rangelands: Current Applications and Future Potentials. Environmental Practice 8(03), pp. 159168.

Robinson, S. A., Wasley, J. and Tobin, A. K., 2003. Living on the edgeplants and global change in continental and maritime Antarctica. Global Change Biology 9(12), pp. 1681-1717.

Snavely, N., Seitz, S. M. and Szeliski, R., 2006. Photo Tourism: Exploring image collections in 3D. ACM Transactions on Graphics 25(3), pp. 835.

Snavely, N., Seitz, S. M. and Szeliski, R., 2007. Modeling the World from Internet Photo Collections. International Journal of Computer Vision 80(2), pp. 189-210.

Turner, J., Colwell, S. R., Marshall, G. J., Lachlan-Cope, T. a., Carleton, A. M., Jones, P. D., Lagun, V., Reid, P. a. and Iagovkina, S., 2005. Antarctic climate change during the last 50 years. International Journal of Climatology 25(3), pp. 279294.

Wasley, J., Robinson, S. A., Lovelock, C. E. and Popp, M., 2006. Some like it wet biological characteristics underpinning tolerance of extreme water stress events in Antarctic bryophytes. Functional Plant Biology 33(5), pp. 443.

Zarco-Tejada, P. J., 2008. A new era in remote sensing of crops with unmanned robots. SPIE Newsroom pp. 2-4.

Zhou, G., Ambrosia, V., Gasiewski, A. J. and Bland, G., 2009. Foreword to the Special Issue on Unmanned Airborne Vehicle ( UAV ) Sensing Systems for Earth Observations. IEEE Transactions on Geoscience and Remote Sensing 47(3), pp. 687689. 